\section{Deutscher Schmerzkongress 2012}

\section{Körperrepräsentation bei Schmerzerkrankungen}

\author{
Schmerzerleben und Körperwahrnehmung beeinflussen sich erheb- \\ lich. Ausgeprägte Körperschemastörungen wurden bei Patienten mit \\ Complex Regional Pain Syndrome (CRPS) und mit Phantomschmerzen \\ gefunden. Für andere Schmerzerkrankungen sind diese Zusammen- \\ hänge kaum untersucht. Liegt hier der Schlüssel zu völlig neuen \\ Therapieansätzen?
}

Bestimmt erinnern Sie sich noch an die letzte Spritze beim Zahnarzt. Haben Sie schon einmal darüber nachgedacht, warum sich der betäubte Teil ihrer Lippe viel größer anfühlte als gewohnt? Christian Maihöfner, Erlangen, vermutet, dass es infolge der Deafferenzierung eines bestimmten somatosensorischen Areals zu einer Art kortikalen Überbewertung der sensorischen Signale aus den angrenzenden Afferenzen kommt. Vermutlich sind ähnliche kortikale Reorganisations- phänomene im Spiel, wenn Patienten mit Complex Regional Pain Syndrome (CRPS) ihre betroffene Hand als viel größer wahrnehmen als sie tatsächlich ist. Die Ausprägung dieses Phänomens korreliert mit der Dauer der Erkrankung und mit dem Schweregrad eines begleitenden ipsilateralen Neglects.

Mit der Kombination von Hand-Lateralisationstraining, HandbewegungsImagination und Spiegeltherapie konnte in einer Studie eine deutliche Symptom-

\title{
Psychotherapie ist kein Wundermittel bei chronischen Schmerzen
}

Im Rahmen einer Psychotherapie bei chronischen Schmerzpatienten geht es v.a. darum, das Leiden am Schmerz zu verringern sowie soziale und alltagspraktische Fähigkeiten zu fördern. Wer unter einer solchen Behandlung eine mehr als $50 \%$ ige Schmerzreduktion erfährt, hat größere Chancen, dass dieser Effekt auch nach Behandlungsende noch weiter anhält als bei der Pharmakotherapie. Winfried Häuser, Saarbrücken, warnte jedoch vor überzogenen Erwartungen. Bei vielen Patienten mit somatoformen Schmerzstörungen sei der chronische Schmerz nur die Spitze des Eisbergs multipler psychischer und körperlicher Probleme. Häuser hält dann eine frühzeitige und ausreichend intensive Behandlung für essenziell. Psychosomatische Schmerzexperten stellten vor kurzem in einer Übersichtsarbeit eine "Unterdosierung von Psychotherapie" im Rahmen vieler multimodaler Verfahren fest [Nickel R. et al. Ärztliche Psychotherapie. 2012;
7(2):112-7]. Ähnlich wie bei einer fortgeschrittenen Krebserkrankung, die auf mehrere Chemotherapiekombinationen nicht angesprochen hat, solle man aber laut Häuser bei wiederholt psychotherapieresistenten Schmerzerkrankungen auch einen palliativen Ansatz und den „Verzicht auf die vierte Schmerzpsychotherapie oder fünfte stationäre multimodale Schmerztherapie" in Erwägung ziehen. Eine solche „palliative psychosomatische Grundversorgung" umfasst nach Häusers Vorstellung niedrigfrequente, nicht-beschwerdekongruente Kontakte und die Möglichkeit zur Krisenintervention. Gleichzeitig werden nicht-indizierte Therapien wie hoch dosierte Opioide oder invasive Verfahren und Operationen vermieden. Ein realistisches Ziel ist dabei, das derzeitige soziale und alltagspraktische Funktionsniveau des Patienten zu erhalten oder zumindest deren Verschlechterung zu verlangsamen. Thomas Bißwanger-Heim reduktion erreicht werden [Moseley GL. Pain. 2004;108(1-2):192-8].

\section{Gestörte Körperrepräsentation als Schmerzursache?}

Die beschriebenen Störungen der Körperrepräsentation gehen mit Reorganisationsprozessen in parietalen und prämotorischen Kortexarealen einher. Bei Phantomschmerzen wurden ähnliche Veränderungen beschrieben. Inwiefern diese für neuropathische Schmerzen spezifisch sind, ist unklar. Auch bei Patienten mit chronischen Rückenschmerzen fanden sich neglectähnliche taktile Defizite.

„Der einzige bislang formulierte, stringente Erklärungsversuch für einen solchen Zusammenhang ist die Theorie der sensomotorischen Inkongruenz von McCabe“, erklärt Gerrit Hirschfeld, Datteln. Demnach wird jeder Bewegungsimpuls zusammen mit dem momentanen sensorischen Input - etwa aus den Dehnungsrezeptoren in Muskeln und Sehnen - dazu verwendet, die zukünftige Position des zu bewegenden Körperteils vorauszuberechnen. Diese Vorhersage wird mit dem tatsächlichen sensorischen Input aus den Lagesensoren verglichen. Weichen Vorhersage und aktuelle "Lagemeldung" voneinander ab, führt das laut Theorie zu Missempfindungen bis hin zu Schmerzen [McCabe CS et al. Rheumatology (Oxford). 2005; 44(4):509-16].

\section{Lokalisationsspezifische Defizite}

Hirschfeld und Kollegen fanden nun Hinweise darauf, dass gestörte Körperrepräsentationen bei Schmerzerkrankungen von Kindern und Jugendlichen je nach Lokalisation der Erkrankung auf bestimmte Körperregionen begrenzt sein können. So hatten die Patienten mit chronischen Kopfschmerzen beispielsweise besondere Schwierigkeiten mit der gedanklichen Rotation von Köpfen, nicht aber von anderen Körperteilen oder Gegenständen. Wenn sich Körperschemastörungen bei Schmerzerkrankungen wirklich als lokalisationsspezifisch erwiesen, dann könne man im nächsten Schritt lokalisationsspezifische sensomotorische Übungsprogramme entwickeln und evaluieren, so Hirschfeld.

Thomas Bißwanger-Heim

Bericht vom Deutschen Schmerzkongress 2012, 17.-20. Oktober 2012 in Mannheim 